This item was submitted to Loughborough's Research Repository by the author.

Items in Figshare are protected by copyright, with all rights reserved, unless otherwise indicated.

\title{
Changes in subjective ratings of impulsive steering wheel vibration due to changes in noise level: a cross-modal interaction
}

PLEASE CITE THE PUBLISHED VERSION

http://dx.doi.org/10.1504/IJVNV.2007.014904

PUBLISHER

(C) Inderscience

VERSION

AM (Accepted Manuscript)

LICENCE

CC BY-NC-ND 4.0

\section{REPOSITORY RECORD}

Mansfield, Neil J., J. Ashley, and Andrew N. Rimell. 2019. "Changes in Subjective Ratings of Impulsive Steering Wheel Vibration Due to Changes in Noise Level: A Cross-modal Interaction". figshare. https://hdl.handle.net/2134/12716. 
This item was submitted to Loughborough's Institutional Repository (https://dspace.lboro.ac.uk/) by the author and is made available under the following Creative Commons Licence conditions.

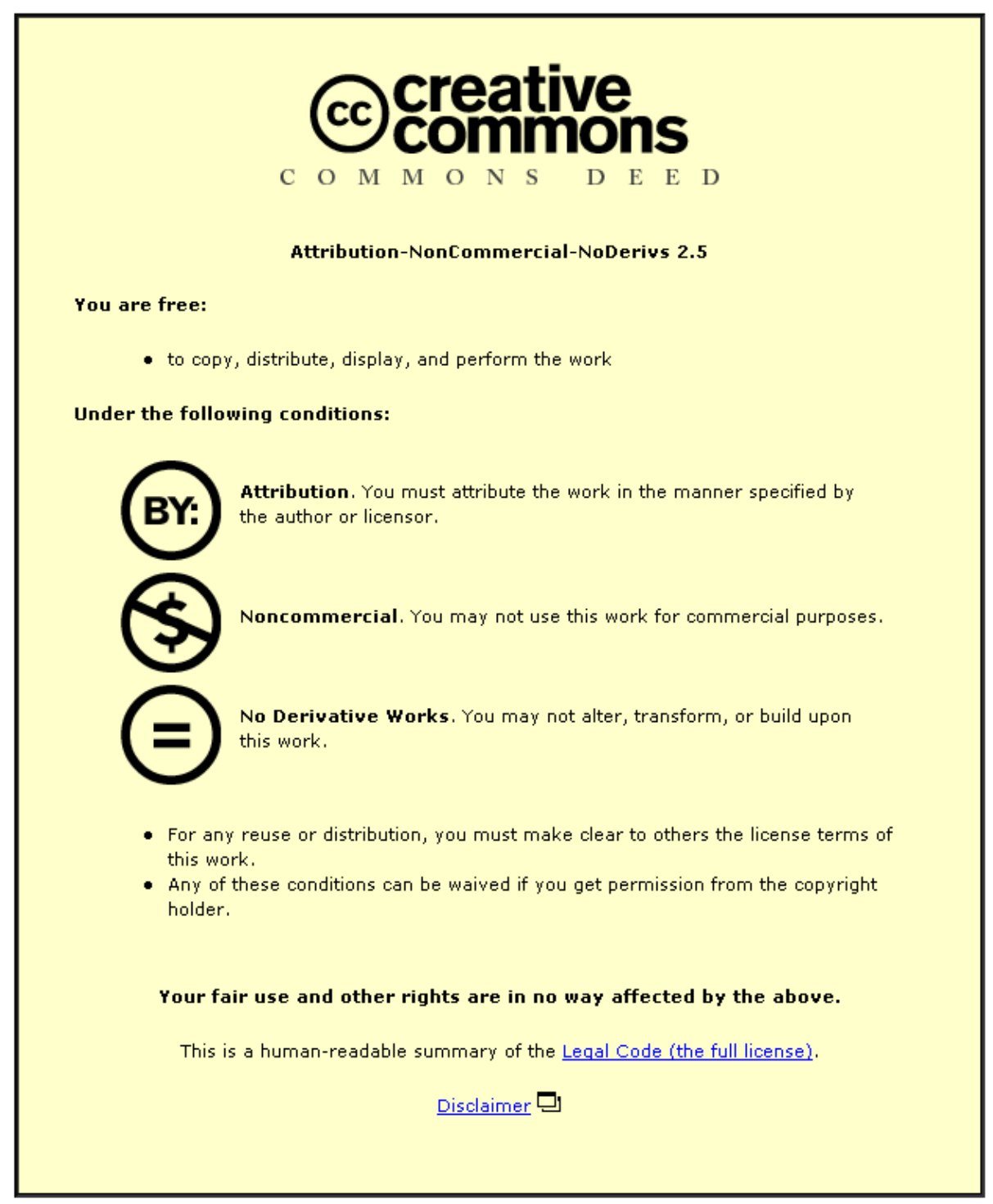

For the full text of this licence, please go to: http://creativecommons.org/licenses/by-nc-nd/2.5/ 
Mansfield, Neil J., Judith Ashley, and Andrew N. Rimell. "Changes in subjective ratings of impulsive steering wheel vibration due to changes in noise level: a cross-modal interaction." International Journal of Vehicle Noise and Vibration 3.2 (2007): 185-196.

http://dx.doi.org/10.1504/IJVNV.2007.014904

\section{TITLE}

Changes in subjective ratings of impulsive steering wheel vibration due to changes in noise level: a cross-modal interaction.

\section{AUTHORS}

Neil J Mansfield, Judith Ashley, Andrew N Rimell

Department of Human Sciences

Loughborough University

Loughborough

Leicestershire

LE11 3TU

n.j.mansfield@lboro.ac.uk

Tel +441509 228483

Fax +44 1509223940 


\section{ABSTRACT}

Cross-modal effects occur when subjective opinions of stimuli from one sense (e.g. tactile at steering wheel) are influenced by simultaneous stimuli in another sense (auditory). A steering wheel rig was used to provide specified vibration stimuli to the participants' hands, and a recording of vehicle sound was played in accordance with the vibrations. Participants were subjected to test conditions with vibration values between 10 and $20 \mathrm{~ms}^{-2}$, and auditory stimuli between 88 and $98 \mathrm{~dB}(\mathrm{~A})$ peak. Participants were neither informed of nor asked about any changes in the noise level: thus the purpose of the experiment was withheld from them. The results show that as noise level increased, subjective ratings of steering wheel vibration increased. Therefore, car cabin noise could be used to enhance the feel of the vibration at the steering wheel.

KEYWORDS Steering wheels, vibration, perception, noise, sound, multi-modal, cross-modal. 


\section{INTRODUCTION}

Drivers are exposed to sensory inputs across multiple modalities simultaneously. They are exposed to noise, vibration, visual stimuli and the thermal environment of the vehicle interior. Each of these modalities can be considered through several tiers of complexity: for example, the thermal environment could be considered in terms of radiant heat, ambient temperature, air velocity, humidity, and the effects of these on whole-body or localised comfort, sweating patterns, etc. assessed (Parsons, 2005). Some types of sensory inputs are desirable (e.g. warning sounds) whilst others might be undesirable (e.g. mechanical shocks induced by road irregularities).

Despite the driving environment comprising stimuli from many modalities simultaneously, most laboratory research has only focused on one modality at a time. Whilst this approach is attractive in terms of experimental simplicity and might possess external validity for examining those factors that are independent (e.g. Hodder and Parsons, 1999), some environmental stressors always occur together and therefore it is possible that in some situations a high degree of experimental ecological validity will be necessary in order for results to possess external validity. For example, subjective responses to precipitation noise could be greatly affected by the effects on vision.

Although driving is primarily a visual task, important sensory information is also perceived through the balance organs, tactile and auditory senses (e.g. 
Haslegrave, 1993; Stanton and Pinto, 2000; El Falou et al., 2003; Giacomin and Woo, 2004; Mansfield, 2005; Mansfield and Whiting-Lewis, 2004; Nakashima and Maeda, 2004). If the mechanical link between the driver and road is removed by, for example, implementation of 'drive-by-wire' technology, then the vehicle designer has scope to enhance the "feel" of the car depending on its instantaneous condition. For example, during motorway driving the noise and vibration could be almost eliminated whereas under more demanding conditions, such as driving on a winding country road, the feel of the road could be fed back to the driver and possibly enhanced. An intermediate option could be to capitalise on the cross-modal aspects of sensory perception (e.g. Spence et al., 1998, 2001; Cockburn and Brewster, 2005), such that vibration in the car cabin could be enhanced using an alternative psychophysical channel, such as the auditory system. A simple example could be devised whereby noises from rumble strips could be enhanced electronically (this idea has already been investigated by some automobile manufacturers).

As noise and vibration are closely linked, it is usual to experience a combination of these senses, rather than any one of them in isolation. Therefore, vibration stimuli will almost always be combined with auditory stimuli. Although in many cases the source of the noise and vibration could be the same (e.g. noise or vibration radiating directly from an engine), in other cases the source of the noise is excited by the vibration, as is apparent in 'squeak and rattle' testing in cars (e.g. Beane et al., 1995). Irrespective of the nature of the source, the tactile and auditory signals are simultaneous. 
The sensations produced by the vibration stimuli that reach the vehicle driver can provide important information regarding the state of the vehicle, but can also produce annoyance and discomfort (Shayaa et al., 2001; Jang and Kook, 2004). Although vehicle design is leading to eliminating noise and vibration completely for the sake of comfort, it must be decided if a certain amount of noise and vibration is necessary in order to be able to drive safely, and what type of noise and vibration this should be. For example, general background noise, created mainly by friction between the tyres and the road, can give an indication of how fast the vehicle is moving. Eliminating the noise may lead to a belief that the vehicle is travelling slower than it actually is, and hence lead to travelling at generally higher speeds. Likewise, eliminating vibration may create an improvement in feeling, but a journey that is too comfortable may lead to driver fatigue and losing concentration.

There have been several studies on the interactions between noise and vibration (Sandover, 1970; Harris and Shoenberger, 1970; Sandover and Champion, 1984; Paulsen and Kastka, 1995; Howarth and Griffin, 1990; Giacomin and Fustes, 2005). Most previous studies have focused on wholebody vibration and/or vibration in buildings. Studies of Howarth and Griffin (1990) and of Paulsen and Kastka (1995) both demonstrated the importance of considering both noise and vibration when assessing community responses to environmental noise. Giacomin and Fustes (2005) applied similar methods for steering wheel noise and vibration, and produced "subjective equivalence" 
curves for continuous (15 s) and impulsive sounds and vibration stimuli. The psychophysical method used a forced choice protocol and subjective equivalence was assumed at the $50^{\text {th }}$ percentile of where sound was considered "more unpleasant" than the vibration.

An alternative approach to the issue of multi-modal stimuli is that of cross-modal interaction whereby stimuli in one modality affects human response to stimuli in another modality. Such cross-modal interactions have primarily been observed between visual and auditory channels. For example, Wade et al. (2006) demonstrated that performance relating to auditory tasks can be affected by impairments in vision. Similarly, Hollier et al. (1999) demonstrated a crossmodal interaction whereby reduction in the quality of just one of the visual or audio streams caused a reduction in the perceived quality of both streams. Using fMRI (functional magnetic resonance imaging) scanners, it can be shown which areas of the brain are dedicated to processing information received from the different physical sensors. When individuals are exposed to multi-modal stimuli (e.g. visuo-tactile and audio-visuo-tactile), brain activity occurs in regions which are not stimulated when each individual modality is presented (Macaluso and Driver; 2005), thus suggesting that certain areas of the brain are dedicated to processing multi-modal stimuli.

It is common for a panel to provide opinion of some aspect of perceived vehicle quality through some form of jury testing. The literature includes practical examples of how individuals might prioritise which aspect of the vehicle 
environment should be prioritised when considering control measures (e.g. comparison of noise and vibration from steering wheels: Giacomin and Fustes, 2005). However, there is little data on the cross-modal interaction whereby driver opinion of one type of environmental stressor could be affected by another type of environmental stressor. This paper reports a study which was designed to investigate multi-modal interactions between tactile sensations of driving over a bump in a car, and auditory signals that are associated with the feel of the bump. It was hypothesised that a cross-modal interaction would be observed whereby the perceived magnitude of vibration at the hands would be affected by the noise. 


\section{Method}

Sixteen subjects participated in the laboratory study that was conducted in the Environmental Ergonomics Laboratories of Loughborough University. All participants were students or staff at Loughborough University, aged between 20 and 24 (mean age 21.75), and had held a driving licence for at least 1 year.

A specially designed steering wheel was used in the study, instrumented with strain gauges to measure grip force (see Figure 1). Signals from an Entran EGAS accelerometer mounted at the top of the steering wheel were conditioned and acquired to computer via anti-aliasing filters. The steering wheel vibration was generated by a Ling V406 vibrator amplified by a Ling PA100E CE amplifier. Sounds were played through a single Mission loudspeaker that was placed directly in front of the participant but obscured behind a some thin acoustically transparent cloth. A calibrated Brüel \& Kjær 2231 Sound Level Meter was placed on a tripod, over the shoulder of the participant, less than $50 \mathrm{~cm}$ away from the right ear in order to measure the noise levels yet remain unobtrusive. The system was controlled by a custom written programme written in LabVIEW.

Figure 1 about here.

Grip force can affect subjective ratings of vibration and therefore required control (Haasnoot and Mansfield, 2004a). Participants gripped the steering 
wheel with a force of $20 \mathrm{~N}$. This force was selected as being the average selfselected force from a previous study performed in the laboratory using the same test rig. When the $20 \mathrm{~N}$ force was correctly applied, the trial was started. Each trial was made up of both noise and vibration stimuli. The vibration stimuli were Gaussian random stimuli with energy band limited to the frequency range of 50 to $100 \mathrm{~Hz}$. Stimuli were single-axis rotational. Each stimulus lasted 1 second and had an exponential taper of $0.2 \mathrm{~s}$. The magnitude of each stimulus was either 10, 15, or $20 \mathrm{~ms}^{-2}$ (unweighted r.m.s.) and the stimuli were separated by a gap of 1 second. In addition to the steering wheel vibration, noise was played through the loudspeaker. The noise comprised a recording of background noise measured in a small car driving on a smooth road mixed with the noise of the same car driving over a bump (Figure 2; Haasnoot and Mansfield, 2002). The level of the noise was adjusted such that the peak level was either 88,93 or $98 \mathrm{~dB}(\mathrm{~A})$. The levels of vibration and noise were monitored throughout the experiment. The timing of the noise of the bump corresponded to the presentation of the vibration. The first stimulus was termed the 'reference'; the second stimulus was termed the 'test'. The test protocol is illustrated diagrammatically in Figure 3. Participants were not informed of the presence of the loudspeaker in the laboratory but were only instructed to consider the vibration (noise was not mentioned in the participant information sheet or instructions).

\section{Figure 2 about here}

Figure 3 about here 
Each participant was exposed to eight different combinations of reference and test vibration and noise (Table 1). The stimuli combinations represented increases and decreases in vibration and noise levels that should be perceivable by the subjects (Flindell, 1998) and was confirmed through a pilot study. The eight different combinations were applied twice. The total of 16 conditions was randomised for each participant. The design of the experiment was such that the majority of sounds heard were the same level (of the 32 sounds used in the trials, 24 peaked at $93 \mathrm{~dB}(\mathrm{~A}))$. This design minimised the risk of participants guessing the true purpose of the experiment which might have led to participants deliberately rating their response to the combination of both stimuli, despite the question only relating to the vibration.

\section{Table 1 about here}

The participants were asked to complete a health screening questionnaire, give written informed consent and were given written instructions about the experiment. The participants were informed that they might leave the experiment at any time without reason. The participants were then asked to sit in the rig seat and adjust it as they would when sat in the driver's position in a car.

The psychophysical method used was that of magnitude estimation. Participants were asked to assign the reference vibration a value of 100 ; the 
test stimuli should then be allocated number according to the relative intensity of steering wheel vibration. For example, if the test vibration intensity was thought to be twice that of the reference vibration, it should be allocated the value of 200 . The participants were instructed to release the steering wheel between each test condition and were given practice stimuli until they were familiar with the equipment and clear on what they were required to do. To minimise potential non-linearities in subjective ratings due to the expected power relationship between magnitude and subjective intensity, and to enable comparison of data from subjects using a different ranges of subjective ratings in their responses, data for each subject was normalised. Normalisation was carried out by calculating the mean and standard deviation for each participant's set of results (Roberts, et al., 2005a, 2005b). Each data point was normalised by subtraction of the mean and division by the standard deviation. Thus, each subject's normalised data had a mean of zero and a standard deviation of unity. Loughborough University ethical committee approved the experiment. 


\section{Results}

Effect of vibration magnitude on the subjective rating of steering wheel vibration

For the trials where there was no change in the level of noise between the reference and test stimuli, the subjective ratings of steering wheel vibration intensity increased with vibration magnitude monotonically for most of the participants (Figure 4). Participant 'f5' showed a slight reduction in subjective rating between the $67 \%$ and $150 \%$ conditions. All other participants showed similar trends whereby the ratings increased with vibration magnitude. Differences between the normalised ratings for the conditions where the noise did not change were significant for each combination of vibration magnitude (paired samples t-test, $p<0.001$ ).

Subjective ratings were similar for male and female subjects. There were no significant differences between the normalised ratings obtained between genders for the conditions where the noise did not change between the reference and the test stimuli (independent samples t-test, $p>0.05$ ).

Figure 4 about here 


\section{Effect of noise level on the subjective rating of steering wheel vibration}

Irrespective of the noise level, subjective ratings of vibration magnitude increased as the vibration magnitude increased for most participants (Figure 4). The only anomaly occurred for participant ' $f 5$ ' who showed a slight reduction in subjective rating between the $67 \%$ and $150 \%$ conditions for the $88 \mathrm{~dB}(\mathrm{~A})$ test stimuli. Differences between the ratings of vibration intensity were significant at the two magnitudes tested for both the $88 \mathrm{~dB}(\mathrm{~A})$ and $98 \mathrm{~dB}(\mathrm{~A})$ noise conditions (paired samples t-test, $\mathrm{p}<0.001$ ).

There was a general trend for the added noise to influence the subjective ratings of the steering wheel vibration. For the $67 \%$ vibration condition, normalised ratings for 12 of the 16 participants were greater for the $93 \mathrm{~dB}(\mathrm{~A})$ condition than for the $88 \mathrm{~dB}(\mathrm{~A})$ condition; similarly normalised ratings for 12 of the 16 participants were greater for the $98 \mathrm{~dB}(\mathrm{~A})$ condition than for the $93 \mathrm{~dB}(\mathrm{~A})$ condition. For the $150 \%$ vibration condition, normalised ratings for 9 of the 16 participants were greater for the $93 \mathrm{~dB}(\mathrm{~A})$ condition than for the $88 \mathrm{~dB}(\mathrm{~A})$ condition; normalised ratings for 12 of the 16 participants were greater for the $98 \mathrm{~dB}(\mathrm{~A})$ condition than for the $93 \mathrm{~dB}(\mathrm{~A})$ condition. This small but consistent trend is clearly observed for mean data (Figure 5).

For some participants, there was a large effect of the noise (e.g. participant ' $f 1$ '), whereas others only showed a small effect (e.g. participant ' $m 8$ ' and 'f8'). Two-way ANOVA showed that the main effect of vibration on the subjective ratings was significant $\left(F_{(1,15)}=158.2, p<0.001\right)$. Similarly the main effect of 
noise on the subjective ratings was also significant $\left(F_{(2,30)}=5.07, p<0.05\right)$. There was no significant interaction between vibration and noise $\left(F_{(2,30)}=2.51\right.$, $p=0.099)$. This means that subjective ratings of vibration are affected by vibration at each noise level and that ratings of vibration are affected by noise at each vibration magnitude. 


\section{Discussion}

The increase in subjective rating of vibration intensity with increased vibration magnitude was expected and is in agreement with all other studies that have been completed in the field (e.g. Haasnoot and Mansfield, 2004b; Morioka, 2004; Giacomin et al., 2004). Therefore, the general methodology is validated according to the published literature.

The previously published study with the most similarity to the current study in terms of stimuli is that of Giacomin and Fustes (2005). In the part of their study which considered transient sounds and vibration, increases in vibration magnitude had a non-linear subjective equivalence with sound, using their adaptation of a cross-modal matching experimental protocol. Nevertheless, Giacomin and Fustes' objectives, method and application of results are fundamentally different to the approach of this study. This study was designed to augment an established programme of work investigating confounding factors for rating of impulsive steering wheel vibration, including the effects of grip force, push force, pull force, impulse envelope, impulse duration, impulse sound and subject gender (e.g. Haasnoot and Mansfield, 2002, 2003, 2004a, 2004b). Thus, the focus of this work was on the cross-modal interaction rather than the subjective equivalence between modalities.

In this experiment, participants were only instructed to rate the relative magnitudes for the reference vibration and the test vibration and no reference was made to sound in any part of the subjects' instructions. Any differences 
between the subjective ratings of vibration for those conditions where the noise also changed could therefore be attributed to a cross-modal effect. The changes in the noise were relatively small (the maximum change during each trial was $+/-5 \mathrm{~dB}(\mathrm{~A})$ ) and therefore any changes in cross modal effects would also be expected to be relatively small. A wider range of noise levels could have been used, but this would have run the risk of alerting the participants to the true nature of the investigation. Despite the modest changes in noise level, a statistically significant change in subjective rating of the vibration was observed. Although averaged data shows clear and consistent effects, individual subject data shows some variability. The extent of the variability is not unusual for this type of psychophysical experiment and highlights the inherent variation in sensitivity and subjective responses to vehicle environments.

In contrast to the results shown in this study, Gescheider and Niblette (1967) showed a masking effect caused by noise on perception of tactile stimuli and vice versa. However, in Gescheider and Niblette's study, the stimuli were short clicks and the greatest masking effects were shown for stimuli that were intense. The results of this experiment covered a small range of values (88 $\mathrm{dB}(\mathrm{A})$ to $98 \mathrm{~dB}(\mathrm{~A})$, and 10 to $20 \mathrm{~ms}^{-2}$ (r.m.s.)) and were statistically significant in their findings. It might be possible that if the intensity of the noise increased further, that, initially, the subjective ratings of vibration would increase accordingly; however, beyond a high threshold, a masking effect could occur where the noise dominated the attention of the participants (Pashler, 1999). 
However, such extreme combinations of stimuli are unlikely to occur in road vehicles.

One purpose of this study was to investigate the possibility for enhancing tactile sensations at the hands using the auditory channel. The results show that there is some scope for tactile enhancement by using sound. Therefore, if additional attention is required for a discrete event, a sound reproduction system in a vehicle could be used to artificially increase the sensation. In some scenarios the auditory channel could be used as a warning (e.g. virtual rumble strips). However, the modality of such warning signals is not important - the purpose of the warning it to alert the driver to some potential danger. This study relates to scenarios where it might be desirable to increase sensation at the hands, without the driver consciously perceiving additional sound. Reinforcing sound could be played during some driving events to enhance driver perception, thus improving driver performance. Further research would be required to identify which elements of the driving task require such reinforcement and the nature that the reinforcement should take.

An additional implication of this study is that improvements in vehicle refinement in terms of reducing the noise or steering wheel vibration will not be fully appreciated by the driver unless the improvements are also accompanied by similar improvements in the other modality. If steering wheel vibration is reduced without a similar noise reduction, the subjective impression of the 
vibration at the steering wheel will not change as much as if the noise was reduced as well. 


\section{Conclusions}

Participants' subjective rating of vibration intensity increases as the vibration magnitude increases. Such a trend was observed for all levels of noise that accompanied the vibration. Noise had an effect on perception of vibration magnitude experienced at a steering wheel. An increase of noise increased the perception of vibration magnitude, while a decrease of noise decreased the perception of vibration. These results show that changes in one modality affect subjective responses to the other modality. Therefore, any improvements in refinement in either noise or vibration should be accompanied by similar improvements of the other for the benefits to be fully appreciated by drivers. Alternatively, the auditory channel could be used to enhance the feel of the car by playing appropriate sounds at appropriate times. 


\section{REFERENCES}

Beane, S.M., Marchi, M.M. and Snyder, D.S., 1995. Utilizing optimized panel damping treatments to improve powertrain induced $\mathrm{NVH}$ and sound quality. Applied Acoustics, 45(2), 181-187

Cockburn, A. and Brewster, S.A., 2005. Multimodal feedback for the acquisition of small targets. Ergonomics 48(9), pp 1129-1150.

El Falou, W., Duchene, J., Grabisch, M., Hewson, D., Langeron, Y. and Lino, F. 2003. Evaluation of driver discomfort during long-duration car driving. Applied Ergonomics, 34(3), 249-255.

Flindell, I.H. 1998. Fundamentals of human response to sound. In Fundamentals of Noise and Vibration, edited by Fahy, F. and Walker, J. EandFN Spon. ISBN 0419241809.

Gescheider, G.A., and Niblette, R.K., 1967. Cross-Modality Masking for Touch and Hearing. Journal of Experimental Psychology, Vol.74, No.3, 313320.

Giacomin, J. and Fustes, F. 2005. Subjective equivalence of steering wheel vibration and sound. International Journal of Industrial Ergonomics, 35, 517-526.

Giacomin, J. and Woo, Y.J., 2004 Beyond comfort: information content and perception enhancement. Journal of the Engineering Integrity Society, Vol. 16, 8-16.

Giacomin, J., Shayaa, M.S., Dormegnie, E., and Richard, L. 2004. Frequency weighting for the evaluation of steering wheel rotational vibration. International Journal of Industrial Ergonomics, 33, 527-541.

Haasnoot, R.A. and Mansfield, N.J. 2002. Vibration magnitudes at the seat and steering wheel whilst driving over small bumps in cars. Paper presented at the 37th United Kingdom Conference on Human Responses to Vibration, held at Department of Human Sciences, Loughborough University, UK, 18 - 20 September 2002.

Haasnoot, R.A. and Mansfield, N.J. 2003. Effect of vibration envelope on relative sensation of vibration on a steering wheel. Paper presented at the 38th UK Conference on Human Response to Vibration Institute of Naval Medicine 17th to 19th September 2003.

Haasnoot, R.A. and Mansfield, N.J. 2004a. Effect of push/pull and grip force on perception of steering wheel vibration. Paper presented at the 10th International Conference on Hand-Arm Vibration, 7-11 June, 2004, Las Vegas, Nevada, USA. 
Haasnoot, R.A. and Mansfield, N.J. 2004b. Perception of steering wheel vibration: Effect of magnitude and duration. 39th Meeting of the UK Group on Human Response to Vibration, organised by RMS Vibration Test Laboratory, Held at Ludlow Assembly Rooms, 15-17 September 2004

Harris, C.S., and Shoenberger, R.W., 1970. Combined Effects of Noise and Vibration on Psychomotor Performance. AMRL-TR-70-14 May 1970. Aerospace Medical research Laboratory.

Haslegrave, C.M., 1993. Visual aspects in vehicle design. In Automotive Ergonomics, edited by Peacock, B. and Karwowski, W. Taylor and Francis, London, ISBN 0-7484-0005-2.

Hodder, S.G. and Parsons, K.C., 1999. The effect of simulated solar radiation intensity on human thermal sensation, 99A4082, ATA conference, Florence, Italy.

Hollier, M.P., Rimell, A.N., Hands, D.S. and Voelcker R.M. 1999, Multi-modal Perception. BT Technology Journal 17(1), 35-46.

Howarth, H.V.C., and Griffin, M.J., 1990. Subjective Response to Combined Noise and Vibration: Summation and Interaction Effects. Journal of Sound and Vibration 143(3), 443-454

Jang, H.-K., and Kook, D.-Y., 2004. Objective measurement of the start-motion quality of a forklift truck. Applied Ergonomics 35,467-473.

Macaluso, E. and Driver, J., 2005 Multisensory spatial interactions: a window onto functional integration in the human brain. Trends in Neurosciences 28(5), 264-271.

Mansfield, N.J. and Whiting-Lewis, E.J.S., 2004. Low frequency lateral acceleration and subjective ratings of acceleration intensity and driving confidence in production cars. Journal of Low Frequency Noise, Vibration and Active Control, 23(4). 221-230.

Mansfield, N.J., 2005. Human response to vibration. CRC press, Boca Raton, ISBN 0-415-24239-x.

Morioka, M., 2004. Magnitude dependence of equivalent comfort contours for vertical steering wheel vibration. 39th UK Group Conference on Human Response to Vibration, Ludlow, 15th to 17th September 2004,

Nakashima, Y., and Maeda, S., 2004. Effects of seat-back angle and accelerometer height at the seat-back on seat-back $x$ axis r.m.s. acceleration in field experiments according to the ISO2631-1 standard. Industrial Health 42, 65-74.

Parsons, K.C. 2005, Ergonomics assessment of thermal environments. In Evaluation of human work (ed. Wilson, J.R. and Corlett, E.N.), $3^{\text {rd }}$ Edition. Taylor and Francis.

Pashler, H.E., 1999. The psychology of attention. MIT press, Massachusetts. ISBN 0-262-66156-x. 
Paulsen, R., and Kastka, J., 1995. Effects of combined noise and vibration on annoyance. Journal of Sound and Vibration 181(2), 295-314.

Roberts JR, Jones R, Mansfield NJ and Rothberg S, 2005a. Evaluation of impact sound on the 'feel' of a golf shot. Journal of Sound and Vibration 287, 651-666.

Roberts JR, Jones R, Mansfield NJ and Rothberg SJ 2005b. Evaluation of tactile sensations on the 'feel' of a golf shot Journal of Sound and Vibration 285, 303-319.

Sandover, J., 1970. Interactions Between Noise and Vibration Effects. UK Informal Group meeting In HRU, Vibration Meeting - Sept.1970. Paper No.10, Loughborough Sept.1970. Dept. of Ergonomics and Cybernetics, Loughborough University of Technology.

Sandover, J., and Champion, D.F., 1984. Some effects of a combined noise and vibration environment on a mental arithmetic task. Journal of Sound and Vibration 95(2), 203-212

Shayaa, M.S., Giacomin, J., Dormegnie, E., and Richard, L., 2001. Human Perception of Sinusoidal Rotational Steering Wheel Vibration. Presented at the $36^{\text {th }}$ UK Group Conference on Human Response to Vibration, held at Centre for Human Sciences, QinetiQ, Farnborough, UK, 12-14 September 2001.

Spence, C., Nichols, M.E.R., Gillespie, N., and Driver, J., 1998. Cross-modal links in exogenous covert spatial orienting between touch, audition, and vision. Perception and Psychophysics, 1998, 60 (4), 544-557.

Spence, C., Nichols, M.E.R., and Driver, J., 2001. The cost of expecting events in the wrong sensory modality. Perception and Psychophysics 2001, 63 (2), 330-336.

Stanton, N.A. and Pinto, M., 2000. Behavioural compensation by drivers of a simulator when using a vision enhancement system. Ergonomics, 43(9) 1359-1370.

Wade, C., Davis, J., Marzilli, T.S. and Weimar, W.H. 2006, Information processing capacity while wearing personal protective eyewear. Ergonomics, 49(10), 955 - 967. 


\section{CAPTIONS}

\section{TABLE:}

Table 1 Vibration and sound levels used for the reference and test stimuli for the eight trials, each of which was repeated but presented in a balanced random order.

\section{FIGURES:}

Figure 1. Laboratory set-up as used for the trials showing instrumented steering wheel, car seat and sound level meter. The loudspeaker was situated behind the fabric screen, directly behind the steering wheel.

Figure 2. Segment of audio file used to provide the sound stimulus. The level of the sound was either 88,93 or $98 \mathrm{~dB}(\mathrm{~A})$ peak and was selected depending on the trial.

Figure 3. Diagrammatic representation of test protocol. Simultaneous sounds and steering wheel vibrations were presented in trial pairs. After the 'reference' stimulus, the sound level and vibration magnitudes would increase or decrease to provide the 'test' stimulus. After each trial pair, the subject would give a numerical rating of the magnitude of the test vibration in comparison to the reference vibration. No mention was made of the associated sounds during any part of the experiment.

Figure 4. Normalised subjective ratings of vibration magnitude for each of 8 male and 8 female participants exposed to steering wheel vibration and noise at $88 \mathrm{~dB}(\mathrm{~A})(\circ-\circ), 93 \mathrm{~dB}(\mathrm{~A})(-)$, and $98 \mathrm{~dB}(\mathrm{~A})(\square-\square)$ peak.

Figure 5. Mean normalised subjective ratings of vibration magnitude for 8 male and 8 female participants exposed to steering wheel vibration and noise at 88 $\mathrm{dB}(\mathrm{A})(\circ-\circ), 93 \mathrm{~dB}(\mathrm{~A})(-)$, and $98 \mathrm{~dB}(\mathrm{~A})(\square-\square)$ peak. 


\section{TABLES}

Table 1 Vibration and sound levels used for the reference and test stimuli for the eight trials, each of which was repeated but presented in a balanced random order.

\begin{tabular}{|c|c|c|c|c|c|c|}
\hline \multirow{2}{*}{$\begin{array}{c}\text { Trial } \\
\text { number }\end{array}$} & \multicolumn{2}{|c|}{ Reference } & \multicolumn{2}{|c|}{ Test } & $\begin{array}{c}\text { Change in } \\
\text { vibration }(\%)\end{array}$ & $\begin{array}{c}\text { Change in } \\
\text { sound }(\mathrm{dB}(\mathrm{A}))\end{array}$ \\
\hline 1 & 10 & 93 & 15 & 88 & $150 \%$ & -5 \\
2 & 10 & 93 & 15 & 93 & $150 \%$ & 0 \\
3 & 10 & 93 & 15 & 98 & $150 \%$ & 5 \\
4 & 10 & 93 & 20 & 93 & $200 \%$ & 0 \\
5 & 15 & 88 & 10 & 93 & $67 \%$ & 5 \\
6 & 15 & 93 & 10 & 93 & $67 \%$ & 0 \\
7 & 15 & 98 & 10 & 93 & $67 \%$ & -5 \\
8 & 20 & 93 & 10 & 93 & $50 \%$ & 0 \\
\hline
\end{tabular}




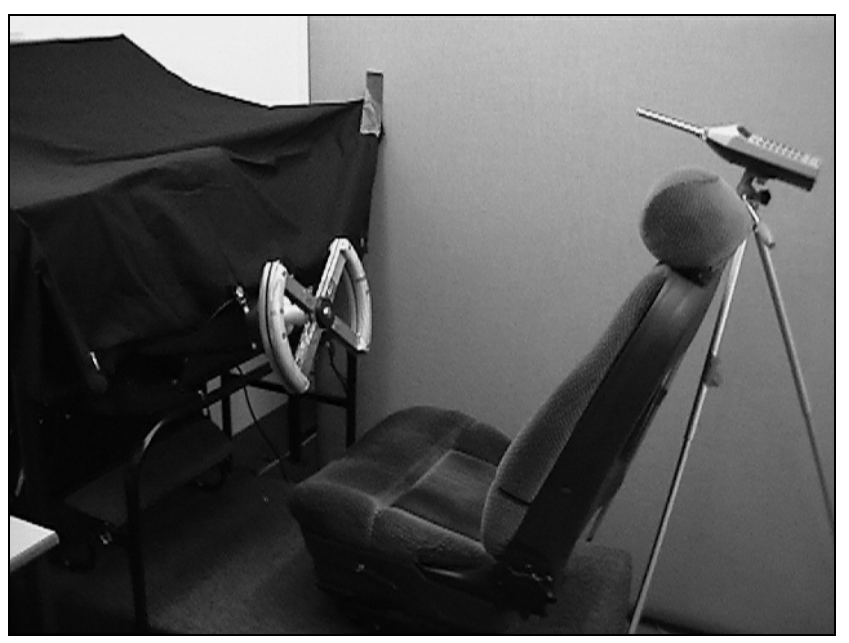

Figure 1. Laboratory set-up as used for the trials showing instrumented steering wheel, car seat and sound level meter. The loudspeaker was situated behind the fabric screen, directly behind the steering wheel. 


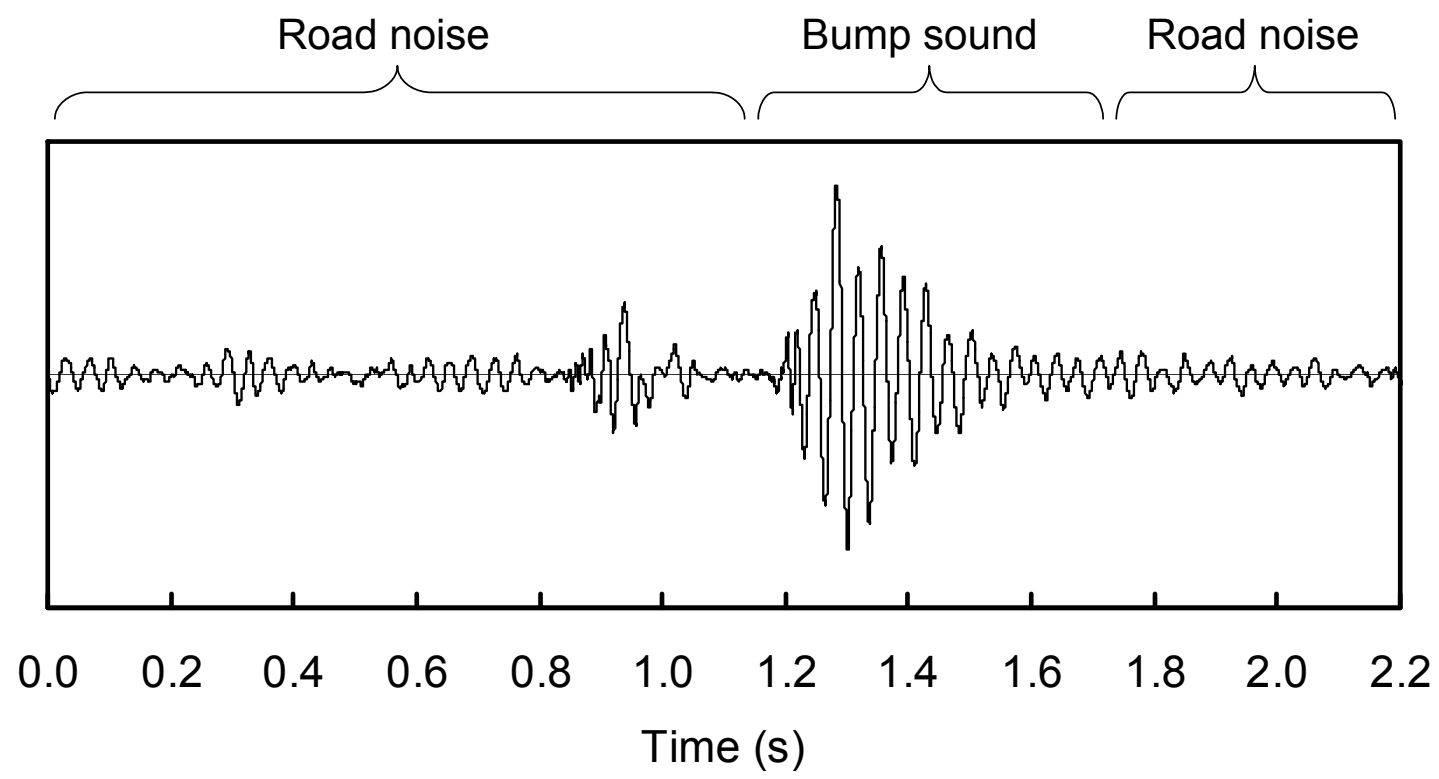

Figure 2. Segment of audio file used to provide the sound stimulus. The level of the sound was either 88,93 or $98 \mathrm{~dB}(\mathrm{~A})$ peak and was selected depending on the trial. 


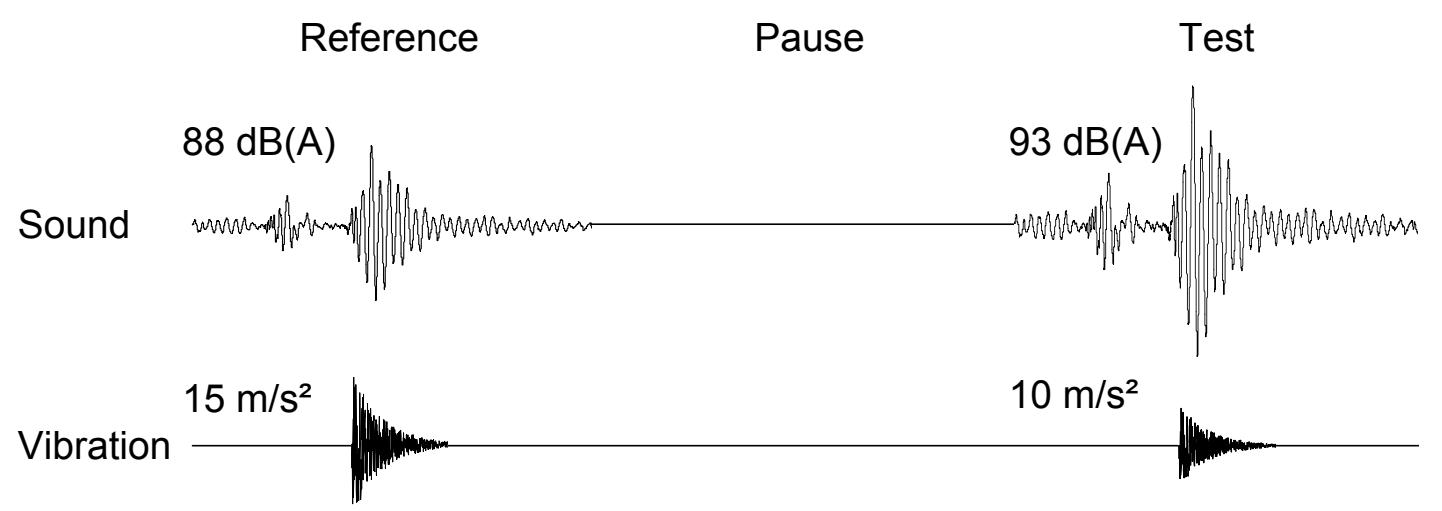

Figure 3. Diagrammatic representation of test protocol. Simultaneous sounds and steering wheel vibrations were presented in trial pairs. After the 'reference' stimulus, the sound level and vibration magnitudes would increase or decrease to provide the 'test' stimulus. After each trial pair, the subject would give a numerical rating of the magnitude of the test vibration in comparison to the reference vibration. No mention was made of the associated sounds during any part of the experiment. 

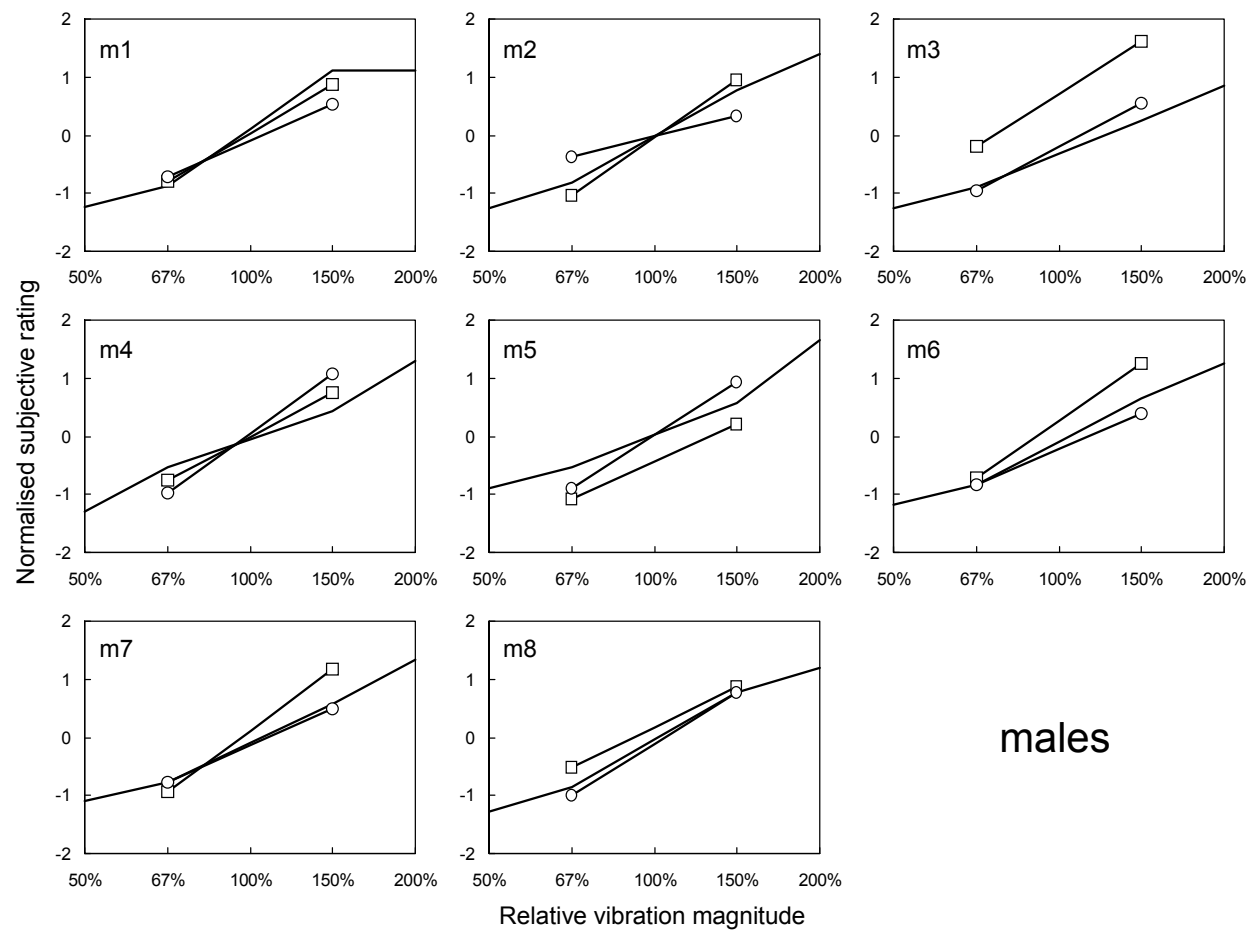

\section{males}
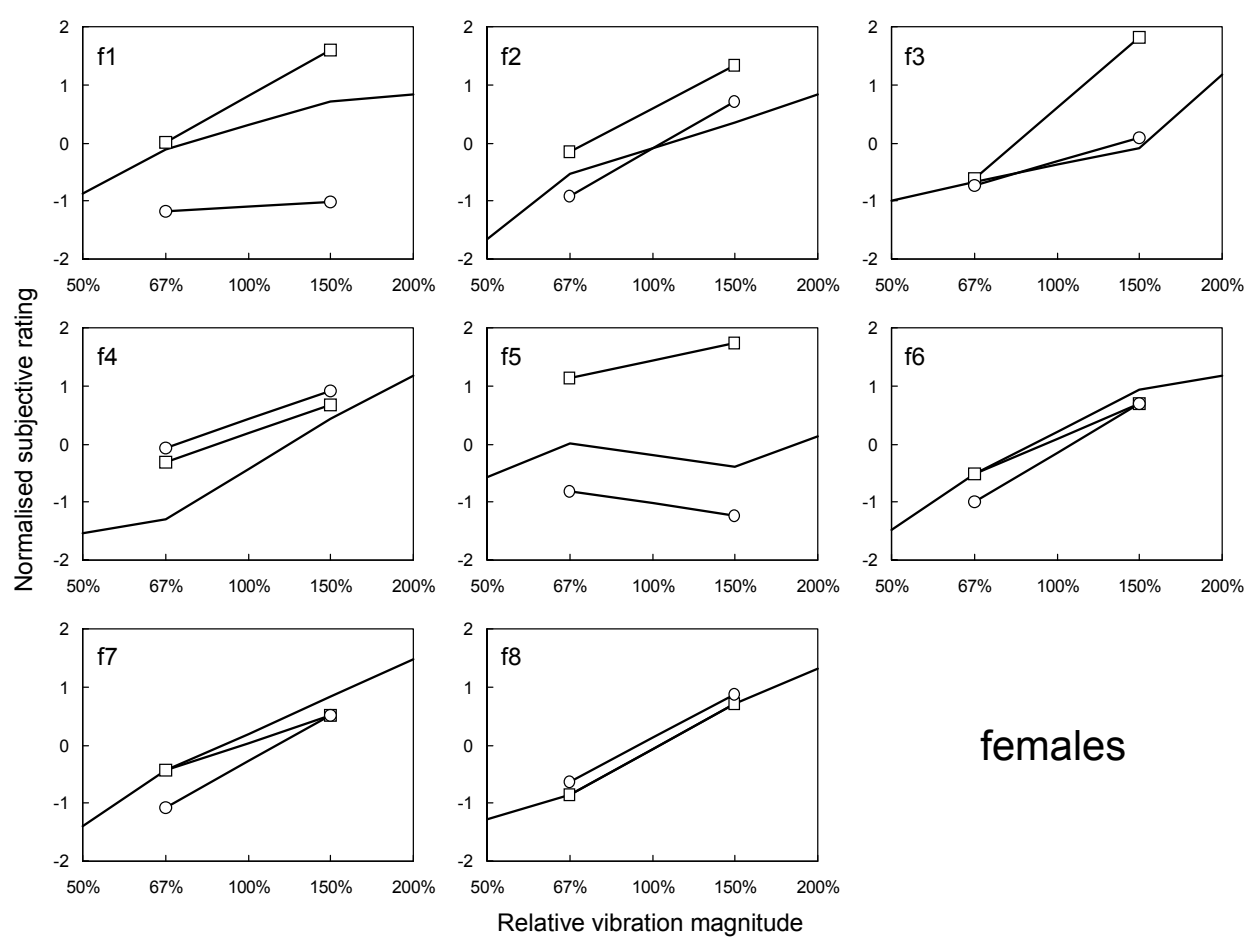

\section{females}

Figure 4. Normalised subjective ratings of vibration magnitude for each of 8 male and 8 female participants exposed to steering wheel vibration and noise at $88 \mathrm{~dB}(\mathrm{~A})(\circ-\circ), 93 \mathrm{~dB}(\mathrm{~A})(-)$, and $98 \mathrm{~dB}(\mathrm{~A})(\square-\square)$ peak. 


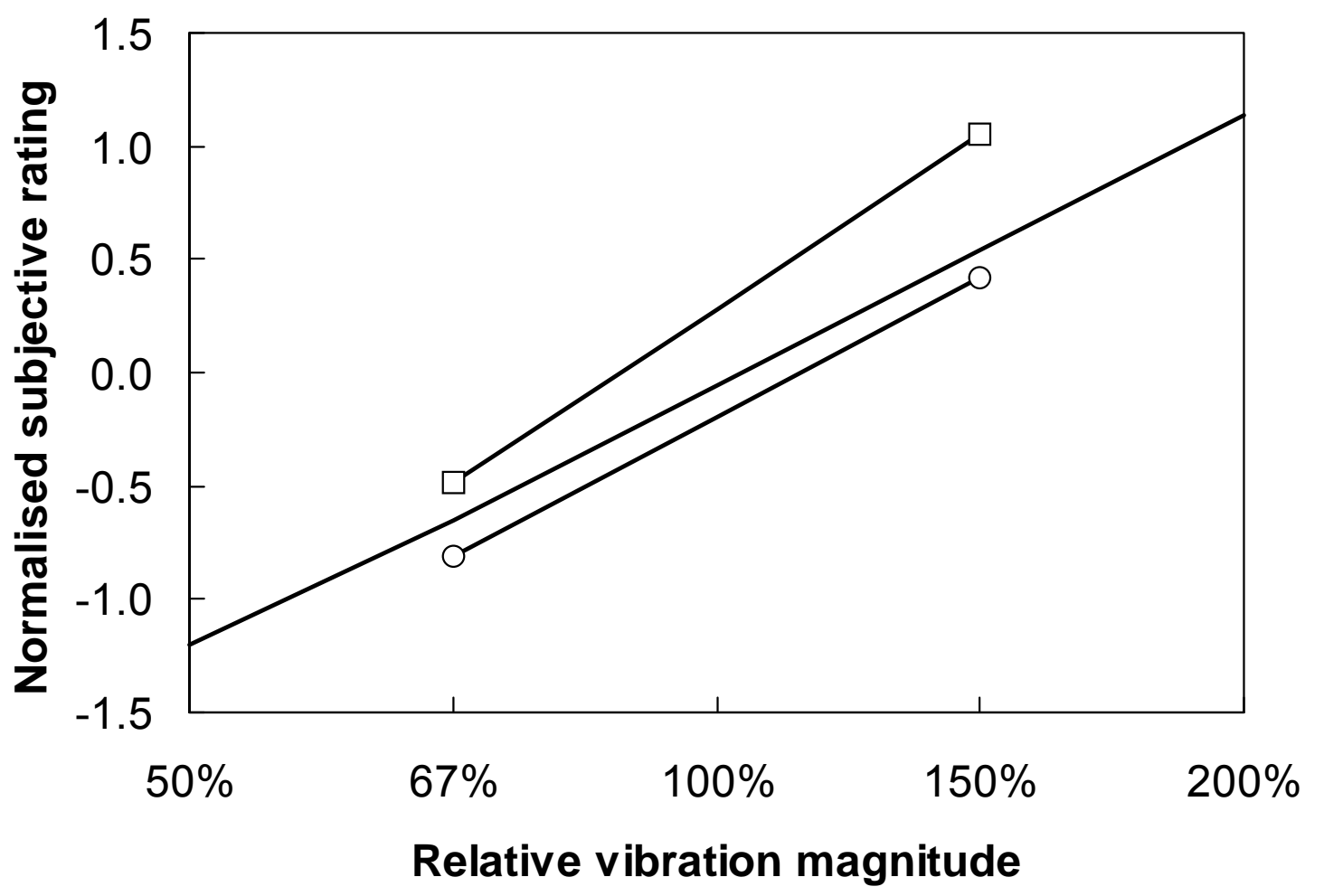

Figure 5. Mean normalised subjective ratings of vibration magnitude for 8 male and 8 female participants exposed to steering wheel vibration and noise at 88 $\mathrm{dB}(\mathrm{A})(\circ-\circ), 93 \mathrm{~dB}(\mathrm{~A})(-)$, and $98 \mathrm{~dB}(\mathrm{~A})(\square-\square)$ peak. 